reading easier. The book predominantly addresses neuroradiologists, neurologists, and emergency department physicians and has a place in the hands of a general practicing physician who wants to better understand the evolving role of CT and MR imaging for triaging patients with acute ischemic stroke. DOI 10.3174/ajnr.A0628

BOOK REVIEW

\section{High Grade Gliomas: Diagnosis and Treatment}

G.H. Barnett, ed. Totowa, NJ: Humana Press; 2006, 495 pages, $\$ 95.00$.

$\mathbf{T}$ his is a good book if you are interested in high-grade gliomas, as many of us are who practice at cancer hospitals. It is the latest offering of the Humana Current Clinical Oncology series. Glioma imaging also happens to be the laboratory in which many new CT, MR imaging, and nuclear medicine techniques are developed and perfected (MR spectroscopy, CT and MR perfusion, and positron-emission tomography), so examining these entities in detail makes sense.

For neuroradiologists, the most interesting parts are the 5 chapters on diagnostic tools, with separate sections on CT, MR imaging, MR spectroscopy, imaging tumor biology, nuclear imaging, and magnetoencephalography. In each there is a focus on relating the imaging findings to the tumor biology, with many chapters authored not only by neuroradiologists but also coauthored by neuropathologists. This gives new meaning to radiographic-pathologic correlation, and it turns out to be important and achievable with every imaging study that can be performed. The ultimate goal seems to be preoperative, in vivo neuropathology. This notion is fully explored in Chapter 8, "Imaging Tumor Biology," with in-depth analysis of just how this in vivo neuropathology will probably emerge from our current anatomic imaging focus.

The rest of the chapters outline the current thinking on all aspects of glioma diagnosis and treatment. One of the most enlightening chapters is the first, "Histologic Classification," which basically confirmed what we had always thought, specifically: even first-rate neuropathologists do not always agree on exactly what to call a tumor on histologic examination. In fact, even the most useful and venerable of the classification schemes, that of the World Health Organization, is subject to constant modification and sometimes even complete revision. Details of classification mechanisms other than anatomic and histologic are included for Ki-67, glial fibrillary acidic protein, epidermal growth factor receptor, and phenotypes. So, in one place, and succinctly presented is information that is particularly pertinent for those who are too impatient to wade through a full neuropathology text. The point is also made, again and again, that specific new imaging techniques increasingly play a part in this overall concept of glioma grading.

New therapy schemes also are part of the text. If you have not yet heard of intracavitary chemotherapy wafers, brachy- therapy, convection therapy, nonconformal stereotactic radiotherapy devices, and all of the new chemotherapy agents, you will someday soon, and this is a good place to find that information. Each chapter is clearly written with a consistent style and not burdened down with technical jargon, but each is up to the minute and authoritative. The book is a recommended addition to the neuroradiologist's reference library.

DOI 10.3174/ajnr.A0626

\section{BOOK REVIEW}

\section{The Physics of Clinical MR Taught Through Images}

V.M. Runge, W.R. Nitz, S. Schmeets, W.H. Faulkner, N.K. Desai, eds. New York: Thieme; 2005, 240 pages, 385 illustrations, $\$ 49.95$.

$\mathbf{T}$ here are a large number of books and journal articles that allow one to gain an appreciation and an understanding of the basics of MR physics. Drs Runge, Nitz, Schmeets, Faulkner, and Desai have added one more to the list, and to this reviewer's eye, The Physics of Clinical MR Taught through Images is certainly one of the quickest and least painful ways of achieving that goal.

In this readily portable, 240-page soft-cover publication, 103 separate topics dealing with the implementation of MR are summarized and accompanied by a few appropriate images for each topic (2 pages per topic). There are many advantages to this approach; one simply has to realize that this is not an in-depth treatise, nor are there references listed. Further reading would be necessary for those desiring more than a basic working understanding of MR physics in their daily work. For most clinical radiologists, however, the information contained in this book would be either adequate or would be a good initial publication from which to build one's knowledge of MR physics.

There is, as one would expect, more than neuro-MR contained in this publication, but neuroradiology images do predominate the pages. Although the sequencing of chapters seem a bit arbitrary (eg, chapters on section thickness or number of averages appear midway through the book, whereas more advanced topics, such as diffusion tensor imaging or blood oxygen level-dependent imaging, precede those more fundamental chapters), one can easily find the item of concern by consulting the table of contents or the index. This book covers hardware (magnets, gradients, and coils), safety (very limited material), newer coil

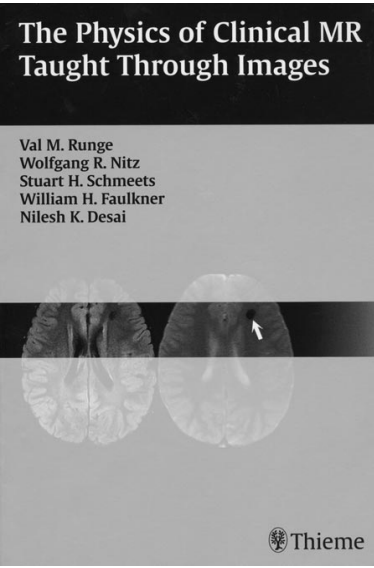

